\title{
Texture Color Fusion Based Features Extraction for Endoscopic Gastritis Images Classification
}

\author{
Zuriani Sobri and Harsa Amylia Mat Sakim
}

\begin{abstract}
Gastritis is a common case when someone suffers a pain or discomfort in the upper part of an abdomen. Conventionally, visual interpretation and pathology diagnosis is employed for identifying abnormalities in a stomach. In this paper, we present a computerized visualization technique which used the image features for classification of endoscopic gastritis image. It is aims to extract features based on texture and color. Gray level co-occurrence matrix (GLCM) features are extracted on wavelet transformed images. Two levels of discrete wavelet transform applied to the antrum image. The texture features then combined with the features of Color Moment for image classification. A support vector machine (SVM) is used as a classifier in categorizing images into its classes. The endoscopic images are classified into normal and abnormal antrum. The combinations of features lead to higher classification rate.
\end{abstract}

Index Terms—Feature extraction, gastritis, SVM, texture.

\section{INTRODUCTION}

Gastritis, also commonly known as heartburn, dyspepsia or acidity, is an irritation or inflammation of the lining in the stomach [1]. It is one of the major digestive problems and up to $10 \%$ of the people who refer to hospital for abdominal pain have gastritis [1], [2]. In 2003, it has been reported that gastric cancer is an important cause of death among patients with malignancies in Malaysia [2]. The inflammation of gastritis is often the effect of Helicobacter Pylori (H. pylori) infection. $\mathrm{H}$. pylori are the bacteria that decline the defensive coating of the stomach and produce a kind of enzyme to irritate the acidity of the stomach lining. Sometimes, gastritis also correlated to other factors such as traumatic injury, regular use of certain pain relievers or drinking too much alcohol.

The standard clinical diagnosis procedure for gastritis is endoscopy, where a thin tube with a tiny light and a camera on the end is inserted down through the throat until the stomach. This procedure allows doctors to observe patients' stomach and take samples called a biopsy to be examined by the pathologist. However, this procedure is considered as invasive, which may cause discomfort to the patient. Furthermore, the conventional procedure requires specialist or experienced doctor. Due to the increasing duty of the

Manuscript received August 30, 2012; September 23, 2012. This research was funded by the Universiti Sains Malaysia (USM) Research University Grant (Grant Number 814082).

Zuriani Sobri is with the Universiti Sains Malaysia, Malaysia (e-mail: zs10_eee058@student.usm.my).

Harsa Amylia Mat Sakim was with Universiti Sains Malaysia. She is now with the School of Electrical \& Electronic Engineering (e-mail: amylia@eng.usm.my). doctors, they only have the limited time to do the task where the priority given to the emergency cases. The others should wait for next appointment to see the specialist or doctors. In fact, early detection of gastritis is crucial. Besides that, the payment cost for the doctors may raise due to extra time required to carry out gastritis diagnosis after working hour. Based on the mentioned problem, this research is intended to provide reliable tools for a computer diagnosis system in order to reduce the additional time required by the specialist. Hence, the extra cost can be avoided, and patients can save their waiting time to be diagnosed.

Due to rapid development of medical image processing, computer-aided diagnosis as well as features extraction techniques has been utilized and become more significant in endoscopic images classification. In 2001, Wang, Krishnan and Tjoa [3] proposed texture features extraction using Local Binary Pattern (LBP) to classify endoscopic images. Neural network classifier results a reasonable classification rate. Tjoa and Krishnan [4] conducted a study to analyses colon status from the endoscopic images using hybrid texture-color features. The Principal Component Analysis (PCA) is employed and average classification accuracy was at $97 \%$. Color wavelet features presented by Karkanis, Iakovidis, Maroulis, Karras and Tzivras [5] to detect tumors in endoscopic videos in 2003. Extraction of color images features using covariance of the second order statistical measure computed over wavelet frame transformation of different color spaces were employed. In 2008 Lima et al. [6] presents an approach to detect abnormality lesions in capsule endoscopic images by means of Color Wavelet Covariance (CWC). These features are estimated over the second order statistical representation of the wavelet transforms of the color image where the co-occurrence matrices approach has been considered. The classification using Radial Basis Function (RBF) resulted in 95\% specificity and 93\% sensitivity. Huang, Chung and Sheu [7] in 2008 employ color, texture and wavelet features extraction technique for H.pylori related gastric histology classification. The classification results using SVM is $87.3 \%$ accuracies. On top of that, the color based method has been introduced by Zhang and Tai [8] in 2008. Many kinds of color features including the color histogram, the main color, the mean color, the dominant color, the color correlogram and color moments were extracted to retrieve endoscopic images information. Combinations of different color features result to different retrieval efficiency.

The proposed approach is to extract features of endoscopic images based on texture-color features. Extracting features from endoscopic images is essential for getting the features which characterize the properties of the stomach lining. These features are significant to provide a reliable features 
extraction tool as a part of an intelligent diagnosis system of endoscopic gastritis.

\section{RELATED WORK}

The experimental data are collected from Hospital Universiti Sains Malaysia (HUSM). For image acquisition, the medical endoscopy system provided by Olympus is used. We used Evis Exera II video imaging system. The endoscopic images captured by the doctors are used in a personal computer for processing. In this research, image analysis incorporates three main processes. The first step is pre-processing. The second step is features extraction where GLCM features are extracted from discrete wavelet transform (DWT) image. Features extraction stage also involved Color Moment approach. Wavelet GLCM and Color Moment features are combined and will be trained and tested using SVM as a classifier. Fig. 1 illustrates the flow of the methodology.

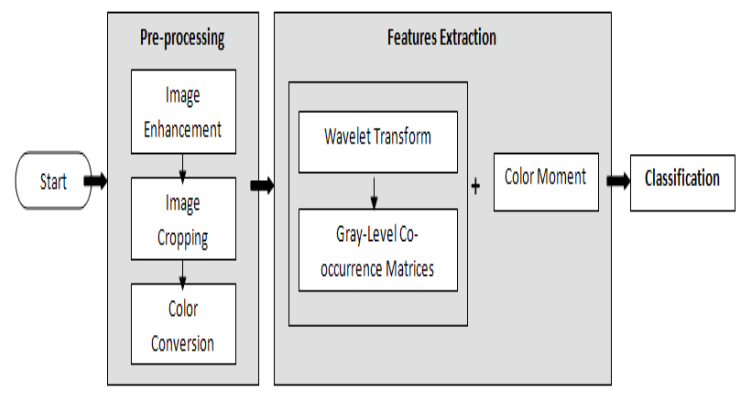

Fig. 1. The flow of methodology

\section{A. Preprocessing}

Pre-processing involved image enhancement, image cropping and image color conversion. This step is the data preparation before the features being extracted. The image enhancement seeks for showing the contrast between redness (gastritis) and its surround antrum lining. In fact, the effect of contrast will lead to a better classification of a normal and abnormal antrum as well as helps in selecting the region of interest (ROI) where the redness can be detected by the naked eyes. Gaussian filter is used for image smoothing and minor noise eliminating. It is can be denoted by (1).

$$
G(x, y)=\frac{1}{2 \pi \sigma^{2}} e^{\frac{-x^{2}+y^{2}}{2 \sigma^{2}}}
$$

where, $G(x, y)$ is the filter mask, $(x, y)$ is the pixels location in the mask window, and $\sigma$ is the standard deviation. We need to produce a discrete approximation to the Gaussian function called kernel before we can perform the convolution. The size of kernel used for this enhancement process is $3 \times 3$. Convolution between kernel and endoscopic image produces a new filtered image, $I_{(x, y)}$. The effect of this method can be seen in Fig. 4. Contrast enhancement is applied to the image

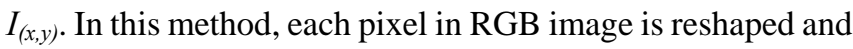
sort in ascending to row vector. $I_{(i, j)}$ is the first pixel in the image while $I_{(m, n)}$ is the last.

$$
R v=\left[I_{(i, j)} \ldots \ldots \ldots \ldots \ldots \ldots I_{(m, n)}\right]^{T}
$$

The formula used for the contrast enhancement is as stated in (3);

$$
G_{(x, y)}=\frac{I_{(x, y)}-\text { threshold }_{\min }}{\text { treshold }_{\max }-\text { treshold }_{\min }}
$$

where treshold $_{\text {min }}$ is obtained by multiplying the minimum weight, $w_{\min }$ with the size of rows, $m$ and columns, $n$ while threshold $_{\max }$ is obtained by multiplying the maximum weight, $w_{\max }$ with the size of $m$ and $n$. Here, the $w_{\min }$ and $w_{\max }$ come from empirical value in the range of 0 to 1 . The most appropriate value for this experiment is 0.09 for $w_{\min }$ and 0.9 for $w_{\max }$. Fig. 5 shows the results of enhancement compared to original image using different values of $w_{\min }$ and $w_{\max }$.

Image cropping is done manually where ROI is cropped to the square of $100 \times 100$ pixels. Experiment has been performed for several sizes of ROIs ( $32 \times 32,64 \times 64$, and $100 \times 100$ pixels) for the same images. A classification result shows that the size of $100 \times 100$ turns out the best results and overspread the uncertainty size of redness on the antrum lining. To choose ROI, the redness must be at the centre of ROI square and it is important to include the background. Thus normal and abnormal region will be discriminated. Once the image has been cropped, it is converted to intensity image since GLCM method is devoted to gray level image. For Color Moment method, endoscopic images are analysed in three types of color spaces, which are RGB, YCbCr and HSV. Each color space has their own strength in highlighting features in the image. They provide a way to identify color that is more intuitive. The three color spaces are split into their single channels, which are R, G, B, H, S, V, Y, Cb and Cr.

\section{B. Discrete Wavelet Transform (DWT)}

DWT acts as a compressor which compresses the processed images more efficiently without sacrificing its clarity. Two dimensional DWT is performed on gray level image because the human visual system responds much more sensitivity to intensity [10]. Fig. 2 shows the structure of two levels wavelet transform.

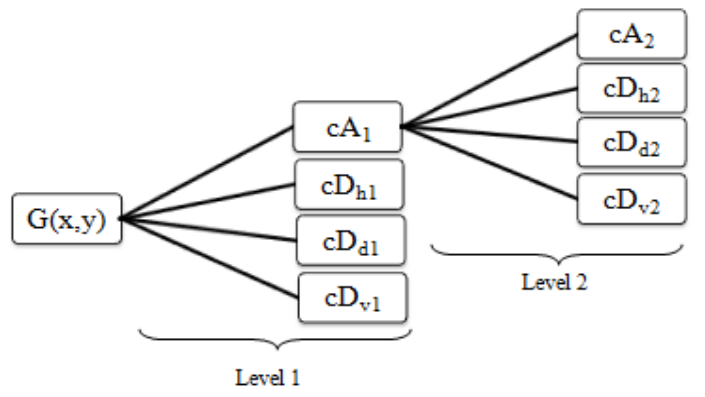

Fig. 2. Structure of wavelet transforms.

$G_{(x, y)}$ is the enhanced image. Applying DWT to $G_{(x, y)}$ generates the coefficient matrices of level one approximation $\left(c A_{1}\right)$, horizontal detail $\left(c D_{h l}\right)$, diagonal detail $\left(c D_{d l}\right)$, and vertical detail $\left(c D_{v l}\right)$. Then, second level decomposition is applied to $c A_{1}$ to generate coefficient matrices of level two approximation $\left(\mathrm{cA}_{2}\right)$, horizontal detail, diagonal detail, and vertical detail $\left(\mathrm{cD}_{\mathrm{h} 2}, c D_{d 2}, c D_{v 2}\right.$ respectively). $c A_{1}$ and $c A_{2}$ is the low-frequency part while others are high frequency. Here, only the coefficient approximations $(c A)$ are considered for 
further processing.

\section{Features Extraction}

Features extraction technique of GLCM is employed for textural information. It is a technique that allows for the extraction of statistical information from the image regarding the pixel pair distributions. GLCM is the most popular method for texture analysis and were demonstrated to feature a potential for effective texture discrimination in biomedical images [4], [9]. GLCM is defined by

$$
P_{d}[i, j]=n_{i j}
$$

where $n_{i j}$ is the number of pixel occurrences. It is computed by defining a direction, $\theta$ and a distance, $d$. Values $(i, j)$ lying at distance $d$ in the image. Then a pairs of pixels separated by $d$, computed across the defined $\theta$ are analysed. As suggested in [12], directions of $0^{\circ}, 45^{\circ}, 90^{\circ}$, and $135^{\circ}$ and the distance of 1 pixel is used in this research. The directions are chosen by assuming that the matrix is symmetrical. Symmetrical means the pixel pair is separated by $\mathrm{d}$ and $-\mathrm{d}$. So, the count will consider the pixel in the direction opposite to $d$. Each pixel pair is computed to get a co-occurrence matrix. Fig. 3(c), (d), (e) and (f) shows matrix frameworks computed from Fig. 3(a).

TABLE I: EQUATIONS FOR GLCM AND COLOR MOMENT FEATURES

\begin{tabular}{|c|c|}
\hline Features & Equations \\
\hline $\mathrm{CON}$ & $\sum_{i} \sum_{j}(i-j)^{k} P_{d}[i, j]^{n}$ \\
\hline \multirow{3}{*}{ CORR } & $\sum_{i} \sum_{j}\left[i j P_{d}[i, j]\right]-\mu_{i} \mu_{j}$ \\
\hline & where $\sigma_{i} \sigma_{j}$ \\
\hline & $\mu_{i}=\sum i P_{d}[i, j], \quad \sigma_{i}^{2}=\sum i^{2} P_{d}[i, j]-\mu_{i}^{2}$ \\
\hline DISS & $\sum_{i} \sum_{j}(i-j) P_{d}[i, j]$ \\
\hline ASM & $\sum_{i} \sum_{j} P_{d}(i, j)^{2}$ \\
\hline ENT & $-\sum_{i} \sum_{j} P_{d}[i, j] \ln P_{d}[i, j]$ \\
\hline IDM & $\sum_{i} \sum_{j} \frac{P_{d}[i, j]}{1+|i-j|^{2}}$ \\
\hline MEAN & $\sum_{i} \sum_{j} \frac{I(x, y)}{m n}$ \\
\hline STD & $\sqrt{\sum_{i} \sum_{j} \frac{I(x-\mu)^{2}}{m n}}$ \\
\hline ENT & $-\sum_{i} \sum_{j} I\left(x_{i}\right) \log _{b} I\left(x_{i}\right)$ \\
\hline SKE & $\sqrt{\sum_{i} \sum_{j} \frac{I(x-\mu)^{3}}{m n}}$ \\
\hline
\end{tabular}

This second-order statistical textural information is calculated over the second level decomposed approximate image. We called it as wavelet-GLCM (WGLCM). From fourteen features introduced by Haralick [12], six features are chosen. Experiment has been done using WEKA machine learning where attributes or features selection applied to select a subset of the features to reduce complexity of computation.

\begin{tabular}{||l|l|l|l|}
\hline \hline 0 & 0 & 1 & 2 \\
\hline 0 & 2 & 2 & 3 \\
\hline 2 & 3 & 3 & 2 \\
\hline 1 & 1 & 2 & 3 \\
\hline
\end{tabular}

(a)

\begin{tabular}{|l|l|l|l|}
\hline 2 & 0 & 0 & 0 \\
\hline 0 & 0 & 1 & 2 \\
\hline 0 & 1 & 6 & 1 \\
\hline 0 & 2 & 1 & 2 \\
\hline
\end{tabular}

(d)

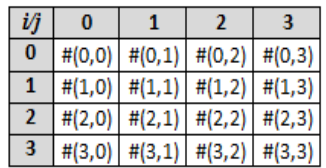

(b)

\begin{tabular}{|l|l|l|l|}
\hline 2 & 1 & 1 & 0 \\
\hline 1 & 2 & 2 & 0 \\
\hline 1 & 2 & 2 & 4 \\
\hline 0 & 0 & 4 & 2 \\
\hline
\end{tabular}

(c)

\begin{tabular}{|l|l|l|l|}
\hline 2 & 0 & 2 & 0 \\
\hline 0 & 0 & 2 & 1 \\
\hline 2 & 2 & 0 & 6 \\
\hline 0 & 1 & 6 & 0 \\
\hline
\end{tabular}

(e)

\begin{tabular}{|l|l|l|l|}
\hline 0 & 0 & 2 & 1 \\
\hline 0 & 0 & 1 & 1 \\
\hline 2 & 1 & 2 & 2 \\
\hline 1 & 1 & 2 & 2 \\
\hline
\end{tabular}

(f)
Fig. 3. (a) Image example, (b) construction of co-occurrence matrix (c) matrix framework for $0^{\circ}$, (d) matrix framework for $45^{\circ}$, (e) matrix framework for $90^{\circ}$, (f) matrix framework for $135^{\circ}$.

From fourteen features, six features (contrast, correlation, dissimilarity, angular second moment, entropy and inverse different moment) are most discriminate. Contrast (CON) is a measure of the local variations present in an image. Correlation (COR) is a measure of image linearity. Correlation will be high if an image contains a considerable amount of linear structure. Dissimilarity (DISS) measures the dissimilarity of image. Angular second moment (ASM) is computed by summing the absolute values in a local neighborhood. Besides that, entropy (ENT) measures the randomness of intensity distribution. Inverse different moment (IDM) will result in a combination of high and low $P[i, j]$ 's in the co-occurrence image.

For Color Moment approach, the features are extracted on the enhanced image. This is because the color features more superior on the enhanced image compared to decomposed image which is more suitable for texture analysis. Color Moment is a simple features extraction technique where four features (mean, standard deviation, entropy and skewness) are extracted from nine color channel (R, G, B, H, S, V, Y, $\mathrm{Cb}, \mathrm{Cr}$ ). Mean (MEAN) gives the average color value for each channel while standard deviation (STD) measures how much the variation or dispersion from the mean value. Skewness (SKE) is a measure of the asymmetry of the data around the sample, and entropy (ENT) is a measure of randomness of color distribution in an image. The equations of GLCM and Color Moment features are stated in Table 1. The total numbers of features based on color are 36 while numbers of features based on texture are 24. All 60 features are combined and write in 60 columns.

\section{SVM Classification}

SVM is one of the kernels based classifier. It is a method of obtaining the optimal boundary of two sets in a vector space independently on the probabilistic distributions of training vectors in the sets. Its fundamental idea is locating the boundary that is most distant from the vectors nearest to the boundary in both classes. For this experiment, we have analysed the data using four types of kernels, which are linear, radial basis function (RBF), quadratic and polynomial to figure out the best kernel for this classification. The total ROI cropped from endoscopic images are 110 ROIs for normal antrum and 110 ROIs for abnormal antrum. All 220 ROIs are classified into two classes, which are normal and abnormal antrum. The condition of images whether it is normal or not has been referred to the pathology department of HUSM. 
These ROIs are equally divided for training and testing. 55 normal ROIs and 55 abnormal ROIs is used for data training while another 55 normal ROIs and 55 abnormal ROIs are for testing.

\section{EXPERIMENTAL RESULTS}

For the experimental result, we performed the effect of image enhancement and the classification results using SVM. Endoscopic image improved in the means of minor noise using Gaussian filter. From Fig. 4(b), we can see that the light reflection from the endoscope over the endoscopic image is dimmed compared to the original image. Fig. 5 represents the contrast enhancement of endoscopic image using three different combination of $w_{\min }$ and $w_{\max }$.

Table III illustrates the classification rate of correctly classified using SVM. Based on the percentage, the combination of GLCM and Color Moment features results to the higher rate which is $87 \%$. This means that out of 110 ROIs, 14 are incorrectly classified. Over four types of kernel used, linear SVM led to a better classification result. This is because linear SVM performs well on data training for features based on Color Moment compared to other kernels. Indirectly, it contributes to higher rate when combining with WGLCM. On top of that, the proposed method has been compared to other traditional texture feature extraction method which has been proposed by Galloway [13]. The method called Gray Level Run Length Matrix (GLRLM). The five features introduced give the highest classification rate of $65 \%$ using polynomial among other kernels. Besides, the straightforward method of gray intensity leads to $85 \%$ using linear SVM, which is still lower compared to proposed approach.

TABLE II: CLASSIFICATION RATE USING SVM

\begin{tabular}{ccccc}
\hline \multirow{2}{*}{ Experiment } & \multicolumn{4}{c}{ Kernel } \\
\cline { 2 - 5 } & linear & RBF & Quadratic & Polynomial \\
\hline $\begin{array}{c}\text { WGLCM }+ \\
\text { Color Moment }\end{array}$ & $87 \%$ & $53 \%$ & $69.4 \%$ & $70 \%$ \\
\hline GLCM & $81 \%$ & $81 \%$ & $82 \%$ & $75 \%$ \\
\hline Color Moment & $85 \%$ & $74 \%$ & $80 \%$ & $75 \%$ \\
\hline GLRLM & $64 \%$ & $61 \%$ & $57 \%$ & $65 \%$ \\
\hline Gray Intensity & $85 \%$ & $50 \%$ & $62.7 \%$ & $61 \%$ \\
\hline
\end{tabular}

\section{CONCLUSION AND FUtURE WORK}

We have presented experimental results describing features extraction of endoscopic image of antrum and the classification using SVM. Our results show that linear kernel of SVM has better performance for this texture-color based features. Choosing the right kernel is the key to improve the performance of the classification. Different kernel may be suitable for difference types of data. Besides that, a good features selection procedure also contributes to higher accuracy of classification. Overall, it is proven that integration of color and texture features extraction for endoscopic images is the best instead of using neither texture nor color independently as been reminded by Tjoa and Krishnan [4]. Currently, we are in the process of expanding the size of data and expect to gain the best result. Furthermore, we will take the challenge to extract more features and develop more appropriate feature selector, which can select the dominant features and reduce the processing time. The size of features will be larger if we used all the approximation and detail coefficients in DWT stage as an input image.

\section{ACKNOWLEDGMENT}

We would like to acknowledge Dr. Lee Yeong Yeah, Dr. Nazri Mustafa, Dr. Nor Aizal Che Hamzah and the staffs of Endoscopy Unit of HUSM for the helps in endoscopic images provision and the guide in medical background.

\section{REFERENCES}

[1] [Online]. Available: Batra's Clinic (Overview-Gastritis), http://www.drbatrsa.com/en/gastritis/

[2] P. Kandasami, W. J. Tan, and K. Norain, "Gastric Cancer in Malaysia: The Need for Early Diagnosis,” Medical Journal of Malaysia, vol. 58, no. 5, pp. 758-762, 2003.

[3] P. Wang, S. M. Krishnan, C. Kugean, and M. P. Tjoa, "Classification of Endoscopic Images Based on Texture and Neural Network," in Proceedings of the $23^{\text {rd }}$ Annual EMBS International Conference, pp. 3691-3695, 2001.

[4] M. P. Tjoa and S. M. Krishnan, "Feature Extraction for the Analysis of Colon Status from the Endoscopic Images,” in Biomedical Engineering Online, pp. 1-5, 2003.

[5] S. A. Karkanis, D. K. Iakovidis, D. E. Maroulis, D. A. Karras, and M. Tzivras, "Computer-Aided Tumor Detection in Endoscopic Video Using Color Wavelet Features," in IEEE Trans. on Information Technology in Biomedicine, vol. 7, no. 3, pp. 141-152, 2003.

[6] C. S. Lima, D. Barbosa, J. Ramos, A. Tavares, L. Monteiro, and L. Carvalho, "Classification of Endoscopic Capsule Images by Using Color Wavelet Features, Higher Order Statistics and Radial Basis Functions," in $30^{\text {th }}$ Annual International IEEE EMBS Conference, pp. 1242-1245, 2008.

[7] C. R. Huang, P. C. Chung, and B. S. Sheu, "Helicobacter Pylori-Related Gastric Histology Classification Using Support-Vesctor-Machine-Based Feature Selection,” in IEEE Trans. On Information Technology in Biomedicine, vol. 12, no. 4, pp. 523-531, 2008.

[8] Q. Zhang and X. Y. Tai, "Endoscope Image Retrieval Based on Color Feature Fusion," Congress on Image and Signal Processing, pp. 247-251, 2008.

[9] M. Kaminishi, H. Yamaguchi, S. Nomura, T. Oohara, S. Sakai, H. Fukutomi, A. Nakahara, H. Kashimura, M. Oda, T. Kitahora, H. Ichikawa, T. Yabana, Y. Yagawa, T. Sugiyama, M. Itabashi, M. Unakami, Y. Oguro, and T. Sakita, “ Endoscopic Classification of Chronic Gastritis Based On A Pilot Study By The Research Society For Gastritis,” Digestive Endoscopic, vol. pp. 14, 138-151, 2002.

[10] R. Lerski, K. Straughan, L. Shad, D. Boyce, S. Bluml, and I. Zuna, "MR Image Texture Analysis - An Approach to Tissue Characterisation,” Magnetic Resonance Imaging, vol. 11, pp. 873-887, 1993.

[11] B. Li and M. Q. H. Meng, "Ulcer Recognition in Capsule Endoscopy Images by Texture Features," in Proceedings of the 7th World Congress on Intelligent Control and Automation, pp. 234-235, 2008.

[12] R. M. Haralick, K. Shanmugam, and I. Dinstein, "Textural Features for Image Classification," in IEEE Trans. On Systems, Man and Cybernatics, vol. 3, no. 6, pp. 610-620, 1973.

[13] M. M. Galloway, “Texture Analsis Using Gray Level Run Lengths,” in Computer Graphics Image Processing, vol. 4, pp. 172-179, 1975.

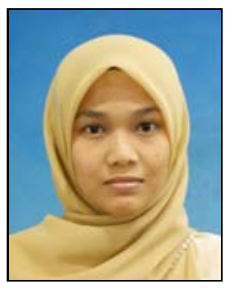

Zuriani Sobri was born in Kedah, Malaysia on February 1984. In 2005 she received her Diploma in Electrical Engineering (Instrumentation) from Universiti Teknologi Mara, Penang, Malaysia. She received Degree in Electrical Engineering from the same university and graduated in 2008. The major field is in electrical system.

She is now a Master student in School of Electrical \& Electronic Engineering, Universiti Sains Malaysia, Penang, Malaysia. Her current research interests include image processing, biomedical engineering and pattern recognition.

Ms. Zuriani is the member of the IEEE from 2009 until now. 


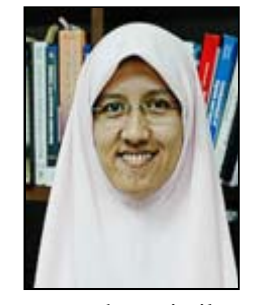

Harsa Amylia Mat Sakim was born in Kuala Lumpur, Malaysia, in November 1971. She received her B.Eng degree from Liverpool University, United Kingdom in July, 1994. She subsequently undertook research at Newcastle University, United Kingdom whom honoured her with an M.Sc degree in 1996. She later pursed her Ph.D degree in breast cancer diagnosis and prognosis based on neural network techniques until February 1998. Subsequently, she pursued her research on similar topics at Universiti Sains Malaysia, whom then honoured her with Ph.D degree. She joined Accurate Information Systems Consultant,
Kuala Lumpur as Associate System Engineer in 1995. In 2003 she joined Universiti Teknologi Mara, Penang as a lecturer. Currently, she is a lecturer at School of Electrical \& Electronic Engineering, Universiti Sains Malaysia. She has published a number of journal and conference paper. Her main interests are in biomedical engineering and cancer research specifically in disease management.

Associate Prof. Dr Harsa Amylia is a member of IEEE, Board of Engineers Malaysia (BEM), and Institute of Engineers Malaysia (IEM). She is the Technology Journal Reviewer and ELEKTRIKA Journal Reviewer of Universiti Teknologi Malaysia since 2005. She is also a member of Malaysian Research and Education Network (MYREN). 